Pediatrics

\title{
Validity evidence of the Brief Fear of Negative Evaluation Scale for children and adolescents adapted for physical activity and sports settings
}

\author{
Glauber Carvalho Nobre $^{1}$ (D) , Nadia Cristina Valentini ${ }^{2}$ \\ ${ }^{1}$ Instituto Federal de Educação Ciência e Tecnologia do Ceará, Departamento de Educação \\ Física, Fortaleza, CE, Brazil. ${ }^{2}$ Universidade Federal do Rio Grande do Sul, Escola de Educação \\ Física, Fisioterapia e Dança, Porto Alegre, RS, Brazil.
}

\begin{abstract}
Associate Editor: Romulo A. Fernandes (D), Universidade Estadual Paulista "Júlio de Mesquita Filho", Presidente Prudente, SP, E-mail: romulo_ef@yahoo.com.br.
\end{abstract}

\begin{abstract}
Aim: To investigate the validity evidence of the adapted Brief Fear of Negative Evaluation Scale in physical activity and sports settings - BFNE-PAS. Methods: Participants included 721 children and adolescents (376 girls and 345 boys), 10 to -14-year-olds (total sample $M_{\text {age }}=12.1, S D=1.3$ ) from Brazil. Results: Exploratory factorial analysis confirmed the unidimensionality of the scale. Confirmatory factorial analysis showed adequate indexes (RMSEA $=0.05,[90 \%$ C.I. $=0.04$ to 0.06$], \mathrm{CFI}=0.98 \mathrm{TLI}=0.98$ ). Network analyses indicate the highest influence of the item: "afraid to expose failures when practicing physical exercises" and/or participating in games and play in physical education classes" upon all others. The polychoric alpha showed adequate internal consistency (total scale: $\alpha=0.86$; items: $\alpha$ values $>0.84)$. The intraclass coefficient correlation showed strong reliability test-retest $($ ICC $>0.90)$. Conclusion: BFNE-PAS showed adequate validity evidence in Brazilian children.
\end{abstract}

Keywords: instruments, childhood, adolescents, social anxiety, network analysis.

\section{Introduction}

The fear of negative evaluation, or social phobia, is a crucial demand related to social anxiety ${ }^{1}$. Individuals with high fear of negative evaluation show exaggerated apprehension when face exposure in social circumstances, avoidance of achievement settings, and frequent feelings of expectation that others will evaluate them negatively ${ }^{2}$. As an aspect of social anxiety, this fear of evaluation from others is related to a range of disruptive behaviors, such as social isolation, difficulty in making friends ${ }^{3}$, and communication in public ${ }^{4}$. Unfavorable health conditions, for example, depressive symptoms ${ }^{5}$ and quality of life $^{6}$ also related to fear of negative evaluation. Besides, specifically, during childhood and adolescence, the fear of negative evaluation affects stronger and undesirably the academic performance and the acquisition of social skills ${ }^{3,7}$.

This fear may occur in various social settings evaluate situations ${ }^{8}$. These require specific performance, such as talking in public and interacting with others ${ }^{9}$. Notably, in physical education and sport contexts, the situations naturally expose the practitioners to social evaluation. Usually, sports practices take place in groups, mediated by a teacher or coach, in settings that allow the evaluation of others (i.e., gymnasium, sports courts, playgrounds, pool). Eventual failure to complete the task satisfactorily is more visible and exposes the practitioners to a social evaluation of their capabilities. Other aspects are related to the use of appropriate dress for physical activity practices (e.g., sports shorts and t-shirts, swimsuits, lightweight t-shirt, or sweatshirts), which may cause distress since it exposes shape or parts of the body. Children and adolescents may be more sensitive to those factors ${ }^{10,11}$. Higher fear of negative social evaluation may lead them to demonstrate high levels of tension, apprehension, and shame of failure during physical activity practices ${ }^{7,12}$. Besides, athletic performance, playing with other children, and physical education classes were reported by children with a social phobia as potentially socially fearful situations ${ }^{7}$. Given this particularity, assessing the fear of negative evaluation and physical education class and sports contexts is essential.

Various self-report scales had been proposed to assess the fear of negative social evaluation in children and adolescents. The Social Phobia and Anxiety Inventory for adolescents ${ }^{13}$; the Social Phobia and Anxiety Inventory for Children ${ }^{14,15}$; the Social Phobia Inventory ${ }^{16}$; Fear of Negative Evaluation scale $^{2}$ are examples of scales that 
assess this construct. Mainly, the Fear of Negative Evaluation scale had been widely utilized in the literature. The original version was developed from an American adult population and contained 30 dichotomous items in a truthfalse format. Posteriorly, Leary ${ }^{17}$ proposed a Brief Fear of Negative Evaluation - BFNE, arguing about the excessive quantity of the item in the original scale. This brief version was composed of 12 items organized in a 5-point scale format rating ranging from 1 (not at all characteristics of me) to5 (extremely characteristic of me. The BFNE showed adequate psychometric properties and has been validated for different adult ${ }^{18-22}$ and adolescent ${ }^{23-25}$ populations.

Although considerable research reports the importance of understanding social anxiety for mental, emotional, and social health in childhood and adolescence, little is known how the fear of negative evaluation interferes with the engagement and permanence of children and adolescences in physical education lessons and motor and sports programs. Nevertheless, this factor is related to avoidance in physical activities, and peers and teammate interactions in sports practices have been understudied. This limitation, in part, is due to the lack of valid and reliable instruments that assess how this construct is manifested in physical education and sports contexts. To the authors' knowledge, no instrument assesses children's and adolescents' fear of negative social evaluation in physical activity and sports settings. Thus, this study aimed to adapt and examine the validity of the Brief Fear of Negative Evaluation Scale for children and adolescents in physical activity and sports settings.

\section{Methods}

\section{Participants}

Participants in the present study included a final sample size of 741 children and adolescents (376 girls; 365 boys), 10 - to -14 -year-olds (total sample Mage $=12.1$, $S D=1.3$; boys: Mage $=12.1, S D=1.3$; girls: Mage $=12.2, S D=1.3$ ) from eight public school in Ceará, Brazil. Children with cerebral paralysis, motor disorder, learning disabilities, and anxiety disorder, reported by parents, teachers, or caregivers, were excluded from the study. Consent was obtained from the custodial caregiver (s), and each child verbally agrees to participate in the study. Three Ph.D. professors, experts in development or sports psychology, and five professionals in motor behavior, sport, and development psychology with five years' experience participated in the face validity. To data analysis the children were randomly allocated in two sample groups as following: 321 children (sample 1: $M=12.1$, $S D=1.2 ; 149$ girls, 46.5\%), 420 children (sample 2: $M=12.2, S D=1.1 ; 220$; girls, $47.6 \%$ ). the scores from sample 1 were used to examine the initial structure of
BFNE- PE. The scores from sample 2 were used in the cross-validation factorial structure. From the total sample, 150 children (age $M=12.2 S D=1.0 ; 75$ girls; $50 \%$ ) were randomly selected to be assessed twice; the scores were used in the test-retest analysis.

\section{Instruments}

The Brief Fear of Negative Evaluation Scale BFNES ${ }^{17}$ was translated and adapted to the present study. The original BFNES is used to determine the degree of apprehension that the individuals experience in a situation in which they are exposed to social evaluation. In doing so, they prospect an unfavorable evaluation from others. The original BFNE is composed of 12 items, being eight specific items and four reverse items, about the fear concerning the lack of approval from others, worries about what kind of impression the individual makes on others, and the worry about saying or doing wrong things. The BFNE is a Likert-type scale from 1 (not at all characteristics of me) to 5 (extremely characteristic of me). The items sum composes a total score, ranging from 12 to 60 . The validity $(r=0.90)$, reliability $(r=0.75)$ and internal consistency $(r=0.96)$ have demonstrated initially for undergraduate students $(17)^{14}$. Psychometric studies of BFNES have been demonstrating adequate evidence of validity and reliability of this scale for children and adolescents $^{19,23}$. In this study, a 5-points Likert scale ("unclear/irrelevant" $=1$; "more or less clear/relevant" $=$ 2 ; "clear/relevant" $=3$; "very clear/relevant" $=4$; "optimal clear/relevant" $=5$ ) was used to assess the expert's judgment about the clarity and relevance of each item of the BFNE.

\section{Procedures}

The ethics committee from proponent University approved the study (CAAE number: 10270118.6.0000.5589). First, four translators participated in the retro translation of BFNE-PAS. Independently, two translators (T1 and T2) with fluency in the original language of the instrument (English) and the target language (Portuguese) translate the scale to Portuguese. An initial Portuguese version was obtained according to the synthesis of these two initial translations. The other two translators (T3 and $\mathrm{T} 4)$ independently conducted the retro translation of the initial version in Portuguese back to the English language. A translator meeting was held, and a final version in Portuguese was obtained by confronting the final Portuguese version with the original version in English.

Since this study proposes an adaptation of the original Brief Fear of Negative Evaluate scale for children and adolescents in physical activity and sport, we consider increasing the construct precision by inserting new items. Although all items from the original version were adapted, we considered that it did not reach the latent construct's 
totality. Thus, three new items were formulated to increase the construct precision for physical activity and sports practice. One item included the worry concerning wearing appropriate clothes (e.g., gym pants, T-shirt, shorts, shorts) in physical activities. One item included the worries about embarrassment or shame caused by a failure in physical activities or sports practices. Lastly, the one new item intended to reinforce the assessment about the fear of exposing failures when practicing physical activity and/or participating in sports matches. The original scale, and the adapted scale, proposed in the present study with three new items, are presented in Table 1.

We contacted the board of education and school administrators from two cities of Ceará, Brazil. Eight public schools responded positively to participation in the study. In a meeting with the school administrator and staff, the researchers explained the study's aims and procedures, and parents were informed about the research. We randomly selected children within the school.

Parents or legal guardians were contacted and received all information about the research procedures; parents' informed consent was obtained from each child participate in this study. The children were assessed individually at schools by trained professionals. The time of the test application was approximately 15 minutes for each participant. The sample size for CFA was estimated, considering a model with 54 degrees of freedom (Number of distinct sample moments - Number of distinct parameters to be estimated: $78-24=54$ ), a significance level at 0.05 , and the desired power at 0.98. Considering a sample loss of $10 \%$, our calculated final sample size for CFA was $\mathrm{n}=420$ participants.

Table 1 - Original scale, and the adapted scale for physical activity and sports.

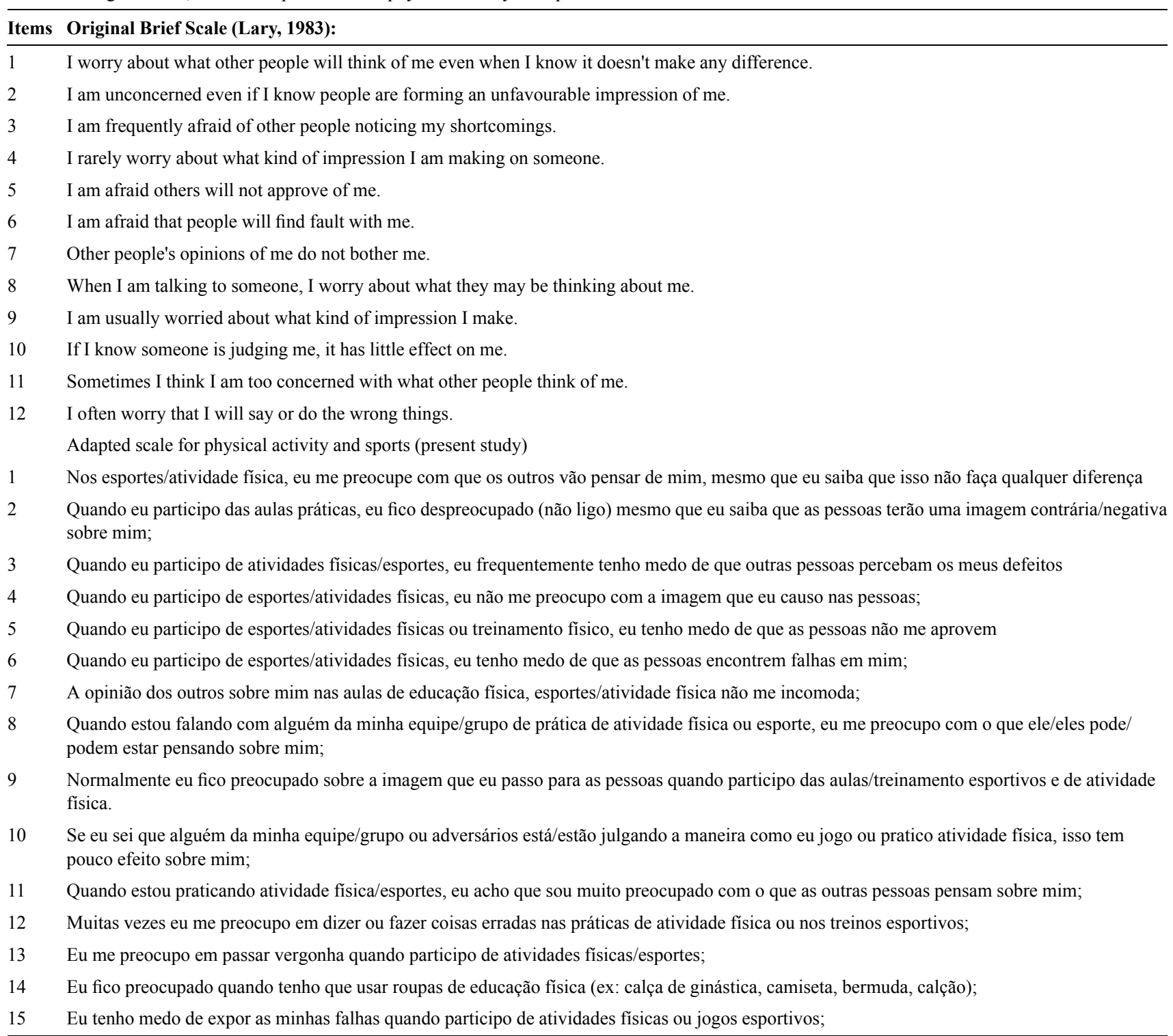


Regarding EFA, we follow the recommended literature of minimum sample size in absolute numbers $\mathrm{n}=$ $300^{26}$. Considering a sample loss of $10 \%$, our calculated sample size for EFA was 330 participants. Considering that nine participants discontinued this study, our final sample size for EFA was 321 children and adolescents.

\section{Statistical Analysis}

Descriptive analysis was provided using mean, standard deviation, and percentages. The Content Validity Coefficient (CVC) was used to estimate each item's language clarity and relevance and total items ${ }^{27}$. The Gwet's Agreement Coefficients (AC1) weighted and unweighted were used to estimate experts' agreement; values above 0.80 were considered a high agreement ${ }^{28}$. Mahalanobis squared distance $\left(D^{2}\right)$ was used to examine multivariate outliers and the Omnibus test based on Small's (chisquare) multivariate nonnormality of the data. We used Exploratory Factorial Analysis (EFA) and Baye's modal estimation polychoric correlation matrices to examine if the new items affected the unidimensional structure ${ }^{29}$. To estimate the adequacy of the correlation matrix, the Kaiser-Meyer-Olkin (KMO) with asymptotic Covariance/Variance matrix, using bootstrap sampling $(95 \%$ confidence interval (CI95\%), and Bartlett's Sphericity Test were conducted. Values higher than 0.80 for KMO and significant statistical differences for Bartlett's test $(p<0.05)$ were considered as adequately. Principal Components extraction method and Promax rotation for EFA analysis and the optimal implementation of Parallel Analysis (PA), to estimate the number of scale dimensions was used ${ }^{30}$.

A confirmatory factorial analysis was conducted to verify the FNE-PE structural model, adopting the weighted least square mean and variance adjusted (WLSMV) estimator's method. The model's overall fit was tested using multiple fit indexes since different measures present strength and weakness ${ }^{31}$. The Tukey Lewis Index (TLI) ${ }^{31}$ and the Comparative Fit Index (CFI); were used to verify model adjustment - values greater than or equal to 0.95 and 0.90 as considered as appropriate ${ }^{32}$. The Root Mean Square Error of Approximation (RMSEA) with $90 \%$ confidence interval (CI90\%), with recognized cut-off ${ }^{32}$; good: values lowest 0.05 ; acceptable: values between 0.06 and 0.08 ) was also used.

The invariance of the model for sex and age (10 to 11 -year-old \& 12 to 14 -year-old) was examined using the multigroup CFA invariance factorial loads. The CFI, GFI, and RMSEA $(90 \% \mathrm{CI})$ were adopted as fit indexes for the configurational invariance. The metric invariance was used to verify if loadings varied across sex and age by groups $^{33}$. Test invariance was used to examine if the intercept for each variable and construct did not vary. The comparisons of the models were conducted using means differences between constrained and unconstrained models, the delta of the RMSEA ( $\triangle$ RMSEA), adopting the recommended cut-off $(<0.015)$ to support the invariance assumption $^{33}$.

Besides, a network analysis was conducted to investigate the structure of partial relationships and the relative influence of items on each other. The estimated Gaussian Graphic Model, with a partial correlation matrix, was used for the network analysis. Thus, the edges represent the conditional association between variables by model, the pairwise Markov field, and it was estimated using the L1regularized neighborhood regression. The regularization was obtained using the graphical Least Absolute Shrinkage and Selection Operator - gLASSO ${ }^{34}$, controlling the model sparsity. Using the Extended Bayesian Information Criteria (EBIC), the model was selected, levels of penalty parameter between 0.25 and 0.50 were adopted. The function "cor_auto" in R-program was used to estimate the correlations matrix. Three centrality measurements were adopted: betweenness, closeness, and strength.

The internal consistency and reliability were conducted to investigate the construct validity using alpha for ordinal data based on polychoric correlations; values $\geq 0.70$ were considered acceptable ${ }^{35}$. Alternatively, the composite reliability (CR) was conducted ${ }^{30}$; CR values equal or superior to 0.70 were considered adequate ${ }^{36}$. In this study, the average variance extracted (AVE) was utilized as a measure of precision. Values superior to 0.50 were considered adequate ${ }^{37}$. The Intraclass Correlation Coefficient (ICC) was utilized to investigate the test-retest reliability. A two-way mixed effect model, based on the mean of multiple measures, was adopted to measure consistency $^{38,39}$.

The analyses were conducted using Agree.15 software, Factor analysis software version 10.9.01, Mplus version 7.4, Psych, and qgraph packages from R-free software. The significance level was set at $\alpha$ less than or equal to 0.05 .

\section{Data sharing}

Data openly is available in a public repository (http://dx.doi.org/10.17632/fcfcyry9mn.1).

\section{Results}

\section{Translation and adaptation}

After the translation and retro translation process, the version of BFNE was adapted to be used in physical activity and sports settings. All items were rewritten (i.e., when I participate in the physical activity and sports, I am frequently afraid of other people noticing my shortcomings; when I am talking to someone from my sport and physical activity team or group, I worry about what they may be thinking about me). Furthermore, three items to the scale: I worry about not being ashamed when I participate in physical education or sports; I get worried when 
I have to wear fitness clothes (gym pants, t-shirt, shorts, shorts); I am afraid of exposing my flaws by doing physical exercises and/or participating in sports matches.

\section{Content validity evidence}

The results showed high CVC values concerning total items among experts for linguistic clarity $(94.7 \%$ to $99.8 \%$ ) and relevance $(96.4 \%$ to $97.5 \%)$. The high CVC results for each item were found (values ranged from $92 \%$ to $100 \%$ for linguistic clarity and $97 \%$ to $100 \%$ for relevance among expert responses). The Gwet's AC1 results ranged from 0.75 to 0.78 for clarity and from 0.84 to 0.93 for relevance, showed high concordance among experts. Table 2 shows the content validity coefficient (CVC) and Gwet's AC1 for language clarity and item relevance.

\section{Construct validity evidence}

The initial EFA analysis enrolled 321 children (sample 1) showed adequate values for $\operatorname{KMO}(0.83, \mathrm{CI} 95 \%=$ 0.81 to 0.85$)$ and Bartlett sphericity test $\left(\chi^{2}(105)=\right.$ 1727.3; $\mathrm{p}<0.001)$. However, were observed inadequate values for load factor $(\lambda<0.50)$ and communalities $(h<0.40)$ in items $2(\lambda=0.12, h=0.01), 4(\lambda=0.37$, $h=0.13), 7(\lambda=0.23 . h=0.05)$, and $10(\lambda=0.34$, $h=0.12$ ). A new EFA, excluding these items, showed adequate suitable for structure detection of the correlation matrix (values for KMO: 0.88 , CI95\% 0.87 to 0.90 ; and Bartlett sphericity test $\left(\chi^{2}(55)=1365.9 ; \mathrm{p}<0.001\right)$. The adequate load factor $(\lambda<0.50)$ and communalities were observed. The model showed adequate adjustment indexes (Weighted Root Mean Square Residual (WRMR) = 0.07, 90C.I. $=0.06 ; 0.08)$. An optimal implementation of Parallel Analysis (PA) and eigenvalue analysis (53\% of explained variance), the Unidimensional Congruence (value $=0.96$; bootstrap $95 \%$ C.I. $=0.94$ to 0.98 ), the Explained Common Variance (value $=0.85$; bootstrap $95 \%$ C.I. $=0.81$ to 0.89 ) and Mean of Item Residual Absolute Loadings $($ value $=0.23$; bootstrap 95\%C.I. $=0.19$ to 0.26 ) indicated a unidimensional scale. The Table 3 provides the factor loadings and communalities values for the BFNE-PE.

To investigate the cross-validation of the BFNE- PE factorial structure, the CFA as conducted with 420 chil- dren (sample 2). The Omnibus analysis, using the Small's test $(\chi 2(24)=542.97 \mathrm{p}<0.001)$, confirmed the multivariate nonnormality of data. A correlation of measurement error between item 3 and item 6 was added to the model, considering the suggestion from modified indexes. The model showed adequate adjustment indexes (RMSEA $=0.05,90 \%$ C.I. $=0.04$ to $0.06, \mathrm{CFI}=0.98 \mathrm{TLI}=0.98)$. Most of the load factors showed values considered adequate $(\lambda>0.50)$, with exception to the item $12(\lambda=0.44)$ and item $14(\lambda=0.50)$. The elimination of these items produced a small modification in the model adjustment $(\mathrm{RMSEA}=0.06$ (90 C.I. $=0.05 ; 0.09$, CFI $=0.96)$. Figure 1 presents the final model of the Fear of Negative Evaluate Scale - short form - adapted for children in physical activity/sports settings.

Was observed, by multigroup analysis, that the model without constriction demonstrated configurational invariance for sex $(\mathrm{CFI}=0.94, \mathrm{RMSEA}=0.004)$ and across age groups $(\mathrm{CFI}=0.95, \mathrm{RMSEA}=0.03)$. Since the loadings do not vary by $\operatorname{sex}\left(\Delta \chi^{2}(10)=11.563 \mathrm{p}=0.315\right.$; $\Delta$ RMSEA $=0.002)$ and by age $\left(\Delta \chi^{2}(10)=7.232\right.$ $\mathrm{p}=0.703 ; \Delta \mathrm{RMSEA}=0.004)$, the metric invariance also was confirmed. Yet, the model indicated that the intercept terms for each variable and construct do not vary by sex $\left(\Delta \chi^{2}(11)=15.408 \mathrm{p}=0.165 ; \Delta\right.$ RMSEA $\left.=0.002\right)$ and age $\left(\Delta \chi^{2}(10)=11.563 \mathrm{p}=0.315 ; \Delta \mathrm{RMSEA}=0.002\right)$.

Table 3 - Factor Loadings and communalities.

\begin{tabular}{lcc}
\hline & Factor loading & $\boldsymbol{h}^{\mathbf{2}}$ \\
\hline Item 1 & 0.70 & 0.50 \\
Item 3 & 0.77 & 0.60 \\
Item 5 & 0.67 & 0.44 \\
Item 6 & 0.73 & 0.53 \\
Item 8 & 0.68 & 0.46 \\
Item 9 & 0.67 & 0.46 \\
Item 11 & 0.79 & 0.62 \\
Item 12 & 0.54 & 0.40 \\
Item 13 & 0.69 & 0.48 \\
Item 14 & 0.65 & 0.42 \\
Item 15 & 0.71 & 0.51 \\
\hline$h^{2}:$ Communalities & &
\end{tabular}

Table 2 - Content validity coefficient (CVC) and Gwet's $\mathrm{AC}_{1}$ concordance coefficient for language clarity and relevance for each item.

\begin{tabular}{|c|c|c|c|c|c|c|}
\hline \multirow[t]{2}{*}{ Experts } & \multicolumn{3}{|c|}{ Clarity } & \multicolumn{3}{|c|}{ Relevance } \\
\hline & $\mathrm{CVC}_{t}(\%)$ & $\mathrm{AC}_{1}(\mathrm{IC} 95 \%)$ & p value & $\mathrm{CVC}_{\mathrm{t}}(\%)$ & $\mathrm{AC}_{1}(\mathrm{IC} 95 \%)$ & $p$ value \\
\hline E-1 $\times$ E- $2 \times$ E- 3 & 99.8 & 0.77 (.42 to 1$)$ & $<0.001$ & 97.5 & 0.91 (.60 to 1$)$ & $<0.001$ \\
\hline $\mathrm{E}-1 \times \mathrm{E}-2$ & 98.7 & 0.75 (.43 to 1$)$ & $<0.001$ & 96.4 & $0.93(.77$ to 1$)$ & $<0.001$ \\
\hline E-1 $\times$ E-3 & 96.8 & $0.75(.43$ to 1$)$ & $<0.001$ & 96.4 & $0.93(.77$ to 1$)$ & $<0.001$ \\
\hline E-2 $\times$ E-3 & 94.7 & $0.78(.45$ to 1$)$ & $<0.001$ & 97.0 & $0.84(.60$ to 1$)$ & $<0.001$ \\
\hline
\end{tabular}

Abbreviation: E1, Expert 1; E2, Expert 2; E3, Expert 3; IC, Interval of Confidence, $\mathrm{AC}_{1}$ - Gwet's Agreement Coefficients; $\mathrm{CVC}_{\mathrm{t}}$ - content validity coefficient for total itens. 


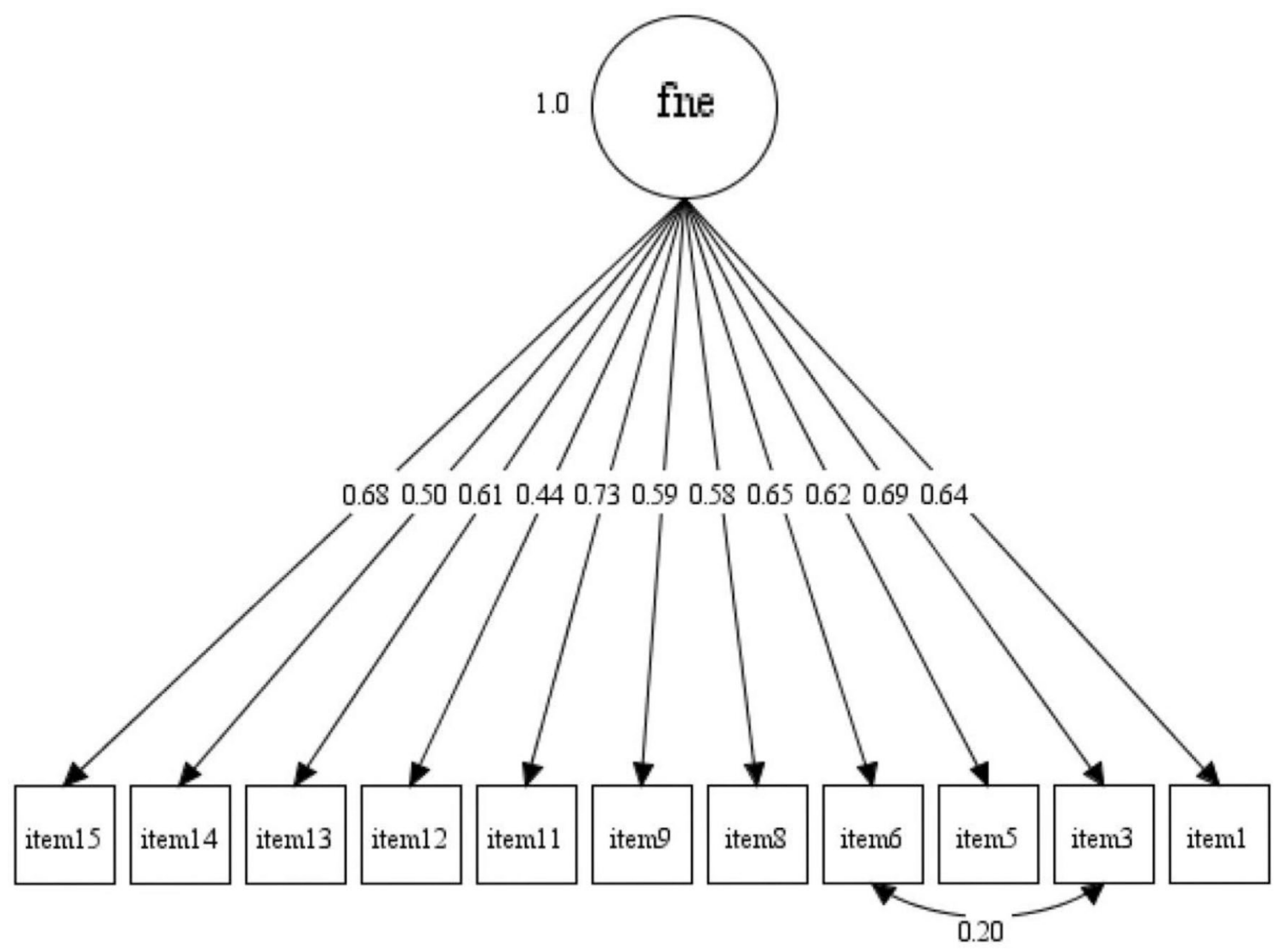

Figure 1 - Factorial structure of the Fear of Negative Evaluate Scale - short form - adapted for children on physical education context.

The network and centrality plot analyses for the BFNE-PAS items were conducted in the total sample (741 children). The highest partial correlations were observed between items 3 and $6(\mathrm{r}=0.26)$, items 9 and 11 $(\mathrm{r}=0.024)$, items 1 and $11(\mathrm{r}=0.20)$ and items 13 and 14 $(\mathrm{r}=0.20)$ The items 11 and 15 showed highest closeness and item 12 and 14 lowest closeness scores in this network analysis. The highest betweenness scores were observed in items 11 and 13. Items 11 and 3 showed the highest strength in the network. The good-of-fit-index showed adequate values for this network (CFI $=1.00$, RMSEA $=0.00$, RMSEA 90C.I. $=0.00 ; 0.01)$. Figure 2 presents the network (Figure 2a) and the centrality plot (Figure $2 \mathrm{~b}$ ) for the items from BFNE-PAS. (a)

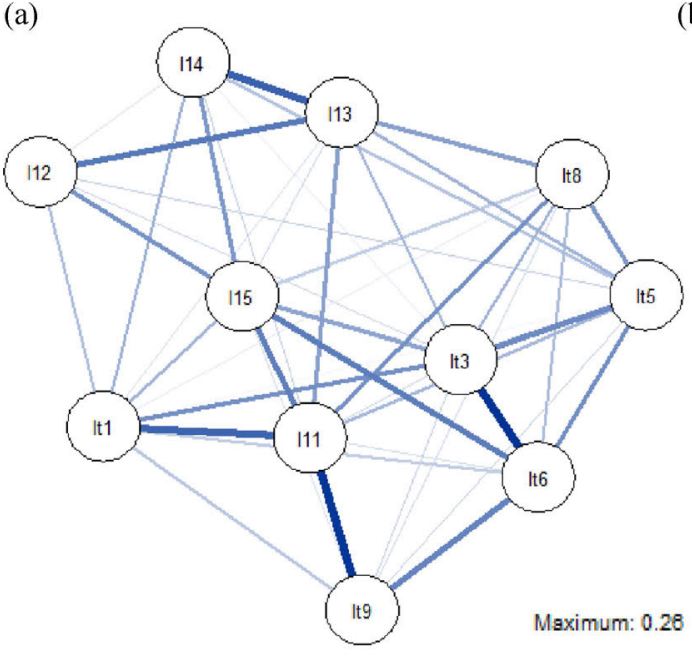

(b)

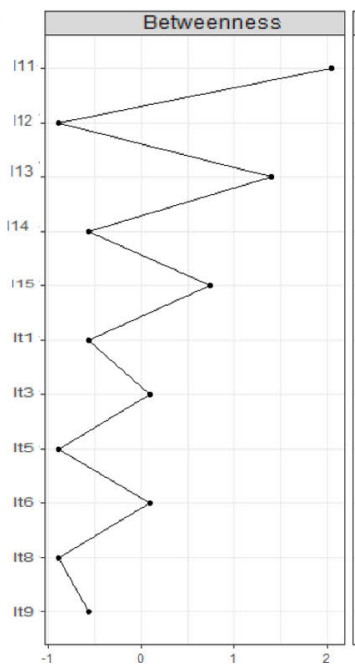

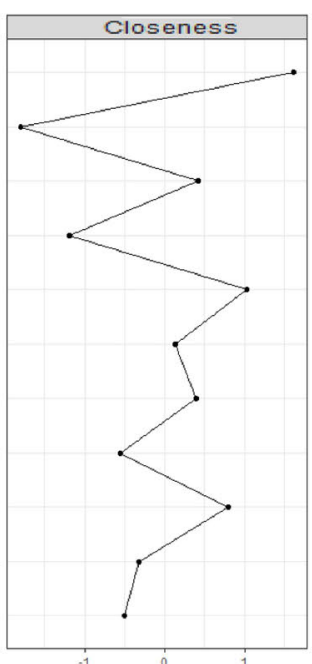

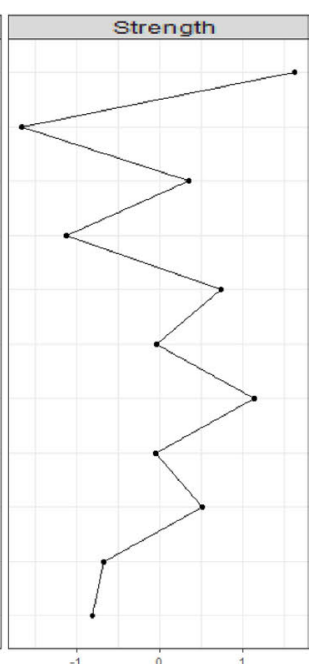

Figure 2 - Network (a) and centrality plot (b) of the items from BFNE-PE. 


\section{Reliability and descriptive analysis}

Table 4 presents the alpha results for ordinal data based on polychoric correlations of the items, the item-rest correlation, and the descriptive statistics (median, minimum, and maximum values) of the scores BFNE-PAS, according to sex and age groups. The results showed an appropriate reliability for all items (values $>0.84$ ) and total scale $(\alpha=0.86)$. In addition, the composite reliability analysis showed adequate value (0.87). Yet, individual item reliability showed appropriate values (values between 0.25 to 0.53 ) except for item 12 (value $=0.19)$. In general, these results reinforcing the internal consistency evidence of the instrument. The AVE value was near to expected (0.40).

Participated of the test- retest analysis, 150 children (age $M=12.2 S D=1.0$ ) (75 girls; $50 \%$ ). Interclass coefficient correlation analysis showed high reliability between test and retest scores for all items (ICC values between 0.90 and 0.93 ; CI $95 \%=0.88 ; 0.96$ ).

\section{Discussion}

This study aimed to examine the validity and reliability of the BFNES-PAS for children and adolescents. After translating and retro translating, a pre-final version was sent to experts. The sentence "I worry about not being ashamed" was replaced by "I worry about embarrassing myself" (item 13). Also, in item 10, the sentence "it has little effect on me" was replaced by "I don't care", following experts' suggestions. This pre-version received strong agreement among experts concerning high or total clarity and relevance ${ }^{27,28}$. Professionals also reported clarity and pertinence of BFNRS-PAS items. The BFNRS-PAS showed adequate content validity evidence.
The initial EFA analysis showed adequate values for $\mathrm{KMO}$ and Bartlett sphericity test, but inadequate load factor $(\lambda<0.50)$ and communalities $(h<0.40)$ values for the items $2,4,7$, and 10 . The items were excluded, and the model showed adequate KMO, Bartlett sphericity, and adjustment indexes ${ }^{30}$. Besides, the version with specific items showed adequate load factor and communalities. The optimal results, in several tests, confirmed the unidimensionality of the scale, namely the implementation of parallel analysis ${ }^{30}$, the congruence, the common variance, and the mean of item residual absolute loadings analyses $^{41}$.

Items 2, 4, 7, and 10 correspond to reverse-worded items from the original short version of the scale. Similarly, other studies reported difficulty in the assessment of the fear of negative evaluation with reverse items. So, compared to the original scale with the brief form that contains only direct items, the original version presented inferior validity indexes ${ }^{18,19}$. For example, in studying Chinese students, the results showed stronger parsimony and excellent reliability and validity only for the BFNE with specific items ${ }^{19}$. Although a two-dimensional model, with one dimension composed only by straightforward items and others with reverse-worded items, has been provided in the literature $20,21,42$ our results provided evidence for unidimensionality of the scale.

The CFA results showed an adequate fit of the mod$\mathrm{el}^{31}$. Most of the load factors showed values considered adequate $^{33}$. Items 12 and 14 showed loads near to adequate, and when eliminated, it produced a small modification in the model. So, for providing additional information to the model, these items were maintained. The multigroup analysis showed configurational, metric, and scalar invariance for sexes and age groups. Therefore, the struc-

Table 4 - Reliability and descriptive statistics for items of the Fear of Negative Evaluation Scale - short form - adapted for children and adolescents for physical activity and sports settings (sample 2).

\begin{tabular}{|c|c|c|c|c|c|c|c|}
\hline \multirow[t]{2}{*}{ Items } & \multirow[t]{2}{*}{ item-rest } & \multirow[t]{2}{*}{$\alpha^{1}$} & \multicolumn{5}{|c|}{ Median values } \\
\hline & & & Boys & Girls & $10-11$ years & $12-14$ years & Total \\
\hline Item 1 & 0.62 & 0.85 & 2 & 2 & 2 & 2 & 2 \\
\hline Item 3 & 0.69 & 0.84 & 2 & 2 & 2 & 2 & 2 \\
\hline Item 5 & 0.60 & 0.85 & 3 & 2.5 & 3 & 2 & 3 \\
\hline Item 6 & 0.67 & 0.84 & 2 & 2 & 2 & 2 & 2 \\
\hline Item 8 & 0.61 & 0.85 & 2 & 2 & 2 & 2 & 2 \\
\hline Item 9 & 0.61 & 0.85 & 3 & 2 & 2 & 2 & 2 \\
\hline Item 11 & 0.73 & 0.84 & 2 & 2 & 2 & 2 & 2 \\
\hline Item 12 & 0.59 & 0.85 & 3 & 3 & 4 & 3 & 3 \\
\hline Item 13 & 0.62 & 0.85 & 3 & 2 & 3 & 2 & 2 \\
\hline Item 14 & 0.66 & 0.85 & 1 & 1 & 1 & 1 & 1 \\
\hline Item 15 & 0.73 & 0.84 & 2 & 2 & 3 & 2 & 2 \\
\hline Total scale & - & 0.86 & - & - & - & - & - \\
\hline
\end{tabular}

Note: $\alpha=$ alpha coefficient; item-rest $=$ polyserial correlation between the item and the sum of the rest of the item scores; ${ }^{1}$ - If item dropped; ${ }^{*}$ Total score from Sum of items. 
ture, including configuration, load factor, and error, did not vary in these groups ${ }^{32}$. Results of an adequate internal structure of BFNE-S were also reported previously ${ }^{21,42}$, similar to our results. Our results for alpha and based on polychoric correlations of the items ${ }^{43}$ and item-total correlations $^{44}$ showed adequate values. The individual item reliability showed appropriate values for most of the items. The composite reliability and $\mathrm{AVE}^{36,44}$ showed fair values and provided additional support for evidence of measure precision ${ }^{37}$ of the BFNES-PE. Also, the intraclass correlation coefficient presents adequate test-retest reliability $^{38,39}$. These results support the high internal consistency.

The network analysis showed adequate good-of-fitindex for relational structure measured. Item 11 showed the highest closeness, betweenness, and strength. Besides, items 15 and 6 showed high closeness values. Closeness, a measure that reveals the shortest path between a given node and the remaining nodes, suggested that these items given a central influence upon others in this network ${ }^{45}$. Betweenness, quantification of the number of times a node acts as a bridge along the shortest path between two other nodes ${ }^{46}$ indicates that, in our data, item 11 seems to mediate the relation between various other nodes in this network. Strength is a measure that indicates a more robust relationship among particular nodes through the sum of the absolute value of its connections with other nodes in a network $^{46}$ suggested that, in our data, the item maintains relationships more robust with other nodes. These results showed that these items have the optimal ability to discriminate between fear of negative evaluation levels in physical education contexts.

These items, especially item 11, are related to what other people think about him/her. It seems that when the judgment from others is more "out of our control" or is less dependent on "own performance", the fear of negative evaluation may be more expressive. So, this is a critical point in the assessment of the fear of negative evaluation. Contrarily, in this network, item 12 showed the lowest closeness, betweenness, and strength. It refers to worry about saying or doing the wrong things, factors that are more within one's control, or depend more on one's attitudes, and therefore were more easily avoidable. Individuals who are high in fear of negative evaluation behave in ways to avoid, when possible, evaluative settings ${ }^{47,48}$. Further, youths with social phobia frequently display a wide range of avoidant strategies when encountering socially distressing situations ${ }^{7}$. These strategies included noncompliance and refusal, such as pretended not to hear the person talking to them, hid their eyes so they would not be called on, and refuse to do as they were asked ${ }^{7}$. So, more avoidable situations may cause less fear of negative evaluation from others.

Explicitly, in physical activity and sports practices, to say or do wrong things depends partly on the individual level, quality of practice, and level of engagement in motor activities. For example, if the children are not required to participate effectively in practices or do practice or do not offer adequate experiences (i.e., challenging tasks), they may choose to avoid the experiences that can fail and consequently receive negative judgment from others. Thus, the feelings of individual control about participation in these situations may affect the fear of negative evaluation from others less strongly. The argument may explain why item 12 showed the lowest influence upon others.

\section{Limitations and strength of the study}

The study's Strength was using robust psychometric procedures in a large and representative Brazilian sample to provide the first validity version of a scale that measures the fear of negative social evaluation in physical activity and sport settings. A limitation of this study was not investigating the criterion validity evidence of the scale. Futures studies can address this unassessed point.

\section{Conclusion}

Based on translation and adaptation procedures and statistical model support, the BFNES - PAS presented substantial evidence of validity to assess the appropriateness of the fear of negative evaluation of Brazilian children in the physical activity and sports settings. This scale could be used to investigate further the complex relationships among other essential aspects, such as engagement in physical activities, self-perceptions of competence, and physical appearance among children and adolescents. Besides, BFNES - PAS's information may help the teachers, coaches, psychologists, and administrators make decisions about the interventional procedures related to social anxiety in sports and physical activity programs.

\section{Declaration of interest statement}

The authors declare no conflicts of interest.

\section{Acknowledgments}

The authors would like to thank all the experts, professionals, as well as children and adolescent participants for their participation and commitment to the study. Further, this study received financial support from Conselho Nacional de Desenvolvimento Científico e Tecnológico (CNPq). N.C.V is Researcher on Productivity.

\section{References}

1. Fredrick JW, Luebbe AM. Fear of positive evaluation and social anxiety: a systematic review of trait-based findings. J Affect Disord. 2020;265(1):157-68. doi 
2. Watson D, Friend R. Measurement of social-evaluative anxiety. J Consult Clin Psychol. 1969;33(4):448-57. doi

3. Beidel DC, Turner SM, Young BJ, Ammerman RT, Sallee FR, Crosby L. Psychopathology of adolescent social phobia. J Psychopathol Behav Assess. 2007;29(1):47-54.

4. Cooper KM, Brownell SE. Student anxiety and fear of negative evaluation in active learning science classrooms. In: Active learning in college science: the case for evidencebased practice. Zürich, Springer; 2020.

5. Kornienko O, Santos CE. The Effects of friendship network popularity on depressive symptoms during early adolescence: moderation by fear of negative evaluation and gender. J Youth Adolesc. 2014;43(4):541-53. doi

6. Dryman MT, Gardner S, Weeks JW, Heimberg RG. Social anxiety disorder and quality of life: how fears of negative and positive evaluation relate to specific domains of life satisfaction. J Anxiety Disord. 2016;38(2):1-8. doi

7. Beidel DC, Turner SM, Morris TL. Psychopathology of childhood social phobia. J Am Acad Child Adolesc Psychiatry. 1999;38(6):643-50. doi

8. Heimberg RG, Brozovich FA, Rapee RM. A cognitivebehavioral model of social anxiety disorder: a update and extension. In: Social Anxiety. Amsterdã, Elsevier; 2010. p. 395-422. doi

9. Furmark T, Tillfors M, Stattin H, Ekselius L, Fredrikson M. Social phobia subtypes in the general population revealed by cluster analysis. Psychol Med. 2000;30(6):1335-44.

10. Trompeter N, Bussey K, Hay P, Mond J, Murray SB, Lonergan $\mathrm{A}$, et al. Fear of negative evaluation and weight/shape concerns among adolescents: the moderating effects of gender and weight status. J Youth Adolesc. 2018;47(7):1398408. doi

11. Pawijit Y, Likhitsuwan W, Ludington J, Pisitsungkagarn K. Looks can be deceiving body image dissatisfaction relates to social anxiety through fear of negative evaluation. Int $\mathbf{J}$ Adolesc Med Health. 2019;31(4):1-7.

12. Ridgers ND, Fazey DMA, Fairclough SJ. Perceptions of athletic competence and fear of negative evaluation during physical education. Br J Educ Psychol. 2007;77(2):339-49. doi

13. Clark DB, Turner SM, Beidel DC, Donovan JE, Kirisci L, Jacob RG. Reliability and validity of the social phobia and anxiety inventory for adolescents. Psychol Assess. 1994;6 (2):135-40.

14. Beidel DC, Turner SM, Morris TL. A new inventory to assess childhood social anxiety and phobia: the social phobia and anxiety inventory for children. Psychol Assess. 1995;7(1):73-9. doi

15. Beidel DC, Turner SM, Hamlin K, Morris TL. The social phobia and anxiety inventory for children (SPAI-C): external and discriminative validity. Behav Ther. 2000;31(1):7587. doi

16. Connor KM, Davidson JRT, Churchill LE, Sherwood A, Weisler RH, Foa E. Psychometric properties of the social phobia inventory (SPIN). Br J Psychiatry. 2000;176(4):37986. doi

17. Leary MR. A brief version of the fear of negative evaluation scale. Personal Soc Psychol Bull. 1983;9(3):371-5. doi
18. Perczel-Forintos D, Kresznerits S. Social anxiety, and selfesteem: hungarian validation of the "Brief Fear of Negative Evaluation scale - straightforward items". Orv Hetil. 2017;158(22):843-50. doi

19. Wei J, Zhang C, Li Y, Xue S, Zhang J. Psychometric properties of the chinese version of the fear of negative evaluation scale-brief (BFNE) and the BFNE-straightforward for middle school students. PLoS One. 2015;10(3):e0115948. doi

20. Tavoli A, Melyani M, Bakhtiari M, Ghaedi GH, Montazeri A. The Brief Fear of Negative Evaluation Scale (BFNE): translation and validation study of the Iranian version. BMC Psychiatry. 2009;9(1):42. doi

21. Koydemir S, Demir A. Psychometric properties of the brief version of the fear of negative evaluation scale in a turkish sample. Psychol Rep. 2007;100(3):883-93. doi

22. Musa C, Kostogianni N, Lépine J-P. The fear of negative evaluation scale (FNE): psychometric properties of the French version. Encephale. 2004;30(6):517-24. doi

23. Karp JN, Makol BA, Keeley LM, Qasmieh N, Deros DE, Weeks JW, et al. Convergent, incremental, and criterionrelated validity of multi-informant assessments of adolescents' fears of negative and positive evaluation. Clin Psychol Psychother. 2018;25(2):217-30. doi

24. Vagos P, Salvador MDC, Rijo D, Santos IM, Weeks JW, Heimberg RG. Measuring evaluation fears in adolescence. Meas Eval Couns Dev. 2016;49(1):46-62. doi

25. Botkin TN, Makol BA, Racz SJ, De Los Reyes A. Multiinformant assessments of adolescents' fears of negative and positive evaluation: criterion and incremental validity in relation to observed behavior. J Psychopathol Behav Assess. 2021;43(1):58-69. doi

26. Tabachnick BG, Fidell LS. Using multivariate statistics. 1st ed. Londres, Pearson; 2013.

27. Hernandez-Nieto R. Contributions to statistical analysis. Mérida, Los Andes University Press; 2002.

28. Gwet KL. Computing inter-rater reliability and its variance in the presence of high agreement. Br J Math Stat Psychol. 2008;61(1):29-48.

29. Choi J, Kim S, Chen J, Dannels S. A comparison of maximum likelihood and bayesian estimation for polychoric correlation using monte carlo simulation. J Educ Behav Stat. 2011;36(4):523-49. doi

30. Lorenzo-Seva U, Timmerman ME, Kiers HAL. The hull method for selecting the number of common factors. Multivariate Behav Res. 2011;46(2):340-64. doi

31. $\mathrm{Hu} \mathrm{L}, \mathrm{Bentler}$ PM. Cutoff criteria for fit indexes in covariance structure analysis: conventional criteria versus new alternatives. Struct Equ Model a Multidiscip J. 1999;6(1):155.

32. Hair JF, Black WC, Babin BJ, Anderson RE. Multivariate data analysis. 7th ed. New York City, Pearson Education; 2010.

33. Kline RB. Principles and practice of structural equation modeling. 3rd ed. London, The Guilford Press; 2011.

34. Friedman J, Hastie T, Tibshirani R. Sparse inverse covariance estimation with the graphical lasso. Biostatistics. 2008;9(3):432-41. 
35. Nunnally JC. Psychometric theory. 3rd ed. Columbus, McGraw Hill Higher Education; 1993.

36. Fornell C, Larcker DF. Evaluating structural equation models with unobservable variables and measurement error. J Mark Res. 1981;18(1):39.

37. Valentini F, Damásio BF. Average variance extracted and composite reliability: reliability coefficients. Psicol Teor e Pesq. 2016;32(2):1-7. doi

38. Qin S, Nelson L, McLeod L, Eremenco S, Coons SJ. Assessing test-retest reliability of patient-reported outcome measures using intraclass correlation coefficients: recommendations for selecting and documenting the analytical formula. Qual Life Res. 2019;28(4):1029-33. doi

39. Shrout PE, Fleiss JL. Intraclass correlations: uses in assessing rater reliability. Psychol Bull. 1979;86(2):420-8.

40. Gwet KL. Variance estimation of nominal-scale inter-rater reliability with random selection of raters. Psychometrika. 2008;73(3):407-30. doi

41. Ferrando PJ, Lorenzo-Seva U. Assessing the quality and appropriateness of factor solutions and factor score estimates in exploratory item factor analysis. Educ Psychol Measur. 2018;78(5):762-80. doi

42. Carleton RN, McCreary DR, Norton PJ, Asmundson GJG. Brief Fear of Negative Evaluation Scale - revised. Depress anxiety. 2006;23(5):297-303. doi

43. Nunnally J. Psychometric methods. New York, McGrawHill; 1978.

44. Bacon DR, Sauer PL, Young M. Composite reliability in structural equations modeling. Educ Psychol Meas. 1995 2;55(3):394-406. doi
45. Borsboom D, Cramer AOJ. Network analysis: an integrative approach to the structure of psychopathology. Annu Rev Clin Psychol. 2013;9(1):91-121. doi

46. Jones PJ, Ma R, McNally RJ. Bridge centrality: a network approach to understanding comorbidity. Multivariate Behav Res. 2021;56(2):353-67-15. doi

47. Essau CA, Conradt J, Petermann F. Frequency and comorbidity of social phobia and social fears in adolescents. Behav Res Ther. 1999;37(9):831-43.

48. Kearney CA. Social anxiety and social phobia in youth: characteristics, assessment, and psychological treatment. Boston, Springer; 2005.

Correspondent author Glauber Carvalho Nobre. Instituto Federal de Educação Ciência e Tecnologia do Ceará, Departamento de Educação Física, Fortaleza, CE, Brazil. E-mail: glauber.nobre@ifce.edu.br.

Manuscript received on June 29, 2021

Manuscript accepted on September 23, 2021

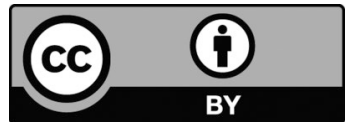

Motriz. The Journal of Physical Education. UNESP. Rio Claro, SP, Brazil - eISSN: 1980-6574 - under a license Creative Commons - Version 4.0 